\title{
Front Matter: Volume 11293
}

, "Front Matter: Volume 11293," Proc. SPIE 11293, MOEMS and Miniaturized Systems XIX, 1129301 (10 April 2020); doi: 10.1117/12.2570451

SPIE. Event: SPIE OPTO, 2020, San Francisco, California, United States 


\title{
PROCEEDINGS OF SPIE
}

\section{MOEMS and Miniaturized Systems XIX}

\author{
Wibool Piyawattanametha \\ Yong-Hwa Park \\ Hans Zappe \\ Editors
}

1-3 February 2020

San Francisco, California, United States

Sponsored by

SPIE

Cosponsored by

Mirrorcle Technologies, Inc. (United States)

Published by

SPIE 
The papers in this volume were part of the technical conference cited on the cover and title page. Papers were selected and subject to review by the editors and conference program committee. Some conference presentations may not be available for publication. Additional papers and presentation recordings may be available online in the SPIE Digital Library at SPIEDigitalLibrary.org.

The papers reflect the work and thoughts of the authors and are published herein as submitted. The publisher is not responsible for the validity of the information or for any outcomes resulting from reliance thereon.

Please use the following format to cite material from these proceedings:

Author(s), "Title of Paper," in MOEMS and Miniaturized Systems XIX, edited by Wibool Piyawattanametha, Yong-Hwa Park, Hans Zappe, Proceedings of SPIE Vol. 11293 (SPIE, Bellingham, WA, 2020) Seven-digit Article CID Number.

ISSN: 0277-786X

ISSN: 1996-756X (electronic)

ISBN: 9781510633490

ISBN: 9781510633506 (electronic)

Published by

SPIE

P.O. Box 10, Bellingham, Washington 98227-0010 USA

Telephone +1 3606763290 (Pacific Time) · Fax +1 3606471445

SPIE.org

Copyright (c) 2020, Society of Photo-Optical Instrumentation Engineers.

Copying of material in this book for internal or personal use, or for the internal or personal use of specific clients, beyond the fair use provisions granted by the U.S. Copyright Law is authorized by SPIE subject to payment of copying fees. The Transactional Reporting Service base fee for this volume is $\$ 21.00$ per article (or portion thereof), which should be paid directly to the Copyright Clearance Center (CCC), 222 Rosewood Drive, Danvers, MA 01923. Payment may also be made electronically through CCC Online at copyright.com. Other copying for republication, resale, advertising or promotion, or any form of systematic or multiple reproduction of any material in this book is prohibited except with permission in writing from the publisher. The CCC fee code is $0277-$ $786 \mathrm{X} / 20 / \$ 21.00$.

Printed in the United States of America by Curran Associates, Inc., under license from SPIE.

Publication of record for individual papers is online in the SPIE Digital Library.

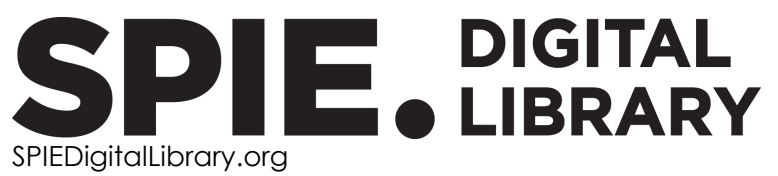

Paper Numbering: Proceedings of SPIE follow an e-First publication model. A unique citation identifier (CID) number is assigned to each article at the time of publication. Utilization of CIDs allows articles to be fully citable as soon as they are published online, and connects the same identifier to all online and print versions of the publication. SPIE uses a seven-digit CID article numbering system structured as follows:

- The first five digits correspond to the SPIE volume number.

- The last two digits indicate publication order within the volume using a Base 36 numbering system employing both numerals and letters. These two-number sets start with $00,01,02,03,04$, 05, 06, 07, 08, 09, OA, OB ... 0Z, followed by 10-1Z, 20-2Z, etc. The CID Number appears on each page of the manuscript. 


\title{
Contents
}

\author{
$\checkmark \quad$ Authors \\ vii Conference Committee
}

MICRO-MIRRORS

$1129302 \quad$ Novel CMOS-integrated 512×320 tip-tilt micro mirror array and related technology platform (Invited Paper) [1 1293-1]

1129303 Design framework for high-speed 3D scanning tools and development of an axial focusing micromirror-based array [11293-2]

$1129304 \quad$ A triple-wafer-bonded AISCN driven quasi-static MEMS mirror with high linearity and large tilt angles [11293-3]

1129305 A bi-axial vacuum-packaged piezoelectric MEMS mirror for smart headlights [11293-4]

LIDAR

1129308 Vibration analysis of micro mirrors for LIDAR using on-chip piezo-resistive sensor (Invited Paper) [11293-7]

1129309 Resonant 1D MEMS mirror with a total optical scan angle of $180^{\circ}$ for automotive LiDAR [1 1293-8]

11293 OB Comparison of MEMS mirror LiDAR architectures [1 1293-31]

NOVEL OPTICAL DEVICES I

11293 OC Wide and fast mode-hop free MEMS tunable ECDL concept and realization in the NIR and MIR spectral regime (Best Paper Award, Invited Paper) [11293-10]

11293 OD Minimization of nonlinearities in nano electrostatic drive actuators using validated coupledfield simulation [1 1293-11]

11293 OF Dynamic pattern generation by singlemode fibers for endoscopic 3D measurement systems [11293-13] 
NOVEL OPTICAL DEVICES II

11293 OG Foveated display by laser scanning [11293-14]

11293 OK Combined MEMS spectrometer based on Michelson interferometer and tunable filter for wideband selective operation [11293-19]

IMAGING

11293 OM Light-sheet microscopy using MEMS and active optics for 3D image acquisition control (Best Student Paper Award, Invited Paper) [11293-21]

$1129300 \quad$ Single-pixel hyperspectral imaging using Hadamard transformation [1 1293-23]

ENDOSCOPIC MICROSCOPY: JOINT SESSION WITH 11214 AND 11293

11293 OR A 3D actuator for laser scanning endoscopy [11293-26]

POSTER SESSION

11293 OS Effects of optically absorbent coatings on parallel optical control of levitating milli-robots [1 1293-17]

11293 OT Compressive sensing MEMS FTIR spectrometer [1 1293-27]

11293 OU Multi-walled carbon nanotubes based near-infrared radiation source [11293-28]

11293 OV Modeling and characterization of the reflectance of vertical metal-coated micromirrors in deeply-etched optical benches [1 1293-29]

11293 OW Attenuated total reflection (ATR) MEMS FTIR spectrometer [11293-30]

11293 OX Coupled MOEMS membranes for enhanced sensing purposes [11293-32]

11293 OY Molybdenum silicide in infrared emitting devices [11293-33]

$11293 \mathrm{OZ}$ System integration of hybrid large aperture micro scanner array for fast scanning LiDAR sensors [1 1293-34]

iv 


\section{Authors}

Numbers in the index correspond to the last two digits of the seven-digit citation identifier (CID) article numbering system used in Proceedings of SPIE. The first five digits reflect the volume number. Base 36 numbering is employed for the last two digits and indicates the order of articles within the volume. Numbers start with 00, 01, 02, 03, 04, 05, 06, 07, 08, 09, OA, OB...0Z, followed by 10-1Z, 20-2Z, etc.

\author{
Albers, Jörg, 05, 09 \\ Altınsoy, Melisa, OR \\ Antipa, Nick, 03 \\ Anwar, Momen, OW \\ Bahr, Andreas, 09 \\ Bakas, Spyridon, OM \\ Baldauf, Julia, OY \\ Baram, A., OG \\ Baver, Ralf, OM \\ Bourouina, Tarik, OU \\ Cahana, G., OG \\ Chau, Fook Siong, 00 \\ Civitci, Fehmi, OR \\ Claus, M., 04 \\ Conrad, Holger, OD \\ Dantan, Aurélien, OX \\ Derman, Irem D., OR \\ Döring, S., 02 \\ Dürr, P., 02 \\ Eckert, M., 02 \\ Ehrig, Lutz, OD \\ ElBidweihy, H., OS \\ Elgner, A., 02 \\ El-Massry, Moez, OV \\ Elsayed, Ahmed A., OU \\ Ersumo, Nathan Tessema, 03 \\ Erten, Ahmet C., OR \\ Ferhanoglu, Onur, OR \\ Fichtner, S., 04 \\ Francés, S., 02 \\ Friedrichs, M., 02 \\ Funck, N., 04 \\ Gabai, R., OG \\ Gehner, A., 02 \\ Ghoname, Amr O., OW \\ Giese, T., 05 \\ Grahmann, Jan, 08, 0C \\ Graßhoff, Thomas, $\mathrm{OZ}$ \\ Gurcuoglu, Oguz, OR \\ Gu-Stoppel, S., 04, 05 \\ Hänsel, L., 02 \\ Heber, J., 02 \\ Hedayet, Karim S., OT \\ Heinrich, Felix, 05, 09 \\ Herrmann, Andreas, $\mathrm{OZ}$ \\ Ho, Derek, OB \\ Hofmann, Ulrich, 05, 09 \\ Hohle, C., 02 \\ Honsberg, Martin, OC
}

Hoppe, Morten, OC

$\mathrm{Hu}$, Frank, OB

Jahns, Daniel, OF

Janicke, Christian, 09

Kaden, Dirk, 09

Kaiser, Bert, OD

Kasturi, Abhishek, OB

Khalil, Diaa A., OK, OT, OU, OV, OW

Khayatzadeh, Ramin, $\mathrm{OR}$

Kiethe, Oliver, 08

Kunze, D., 02

Langa, Sergiu, OD

Li, Liang, 00

Lim, Zi Heng, 00

Lisec, T., 04

Lofink, Fabian, 04, 09

Lopez, Daniel, 03

Lovell, Daniel, OB

Makkapati, S., OS

Malaurie, Pauline, 09

Marty, Frédéric, OU

Melnikov, Anton, OD

Milanović, Veljko, OB

Milde, Tobias, OC

Muller, Rikky, 03

Naesby Rasmussen, Andreas, OX

Naftali, M., OG

Naserbakht, Sepideh, OX

Nazeer, Sebastien, OV

Nefzaoui, Elyes, OU

Nelson, C., OS

Ortlepp, Thomas, OY

Owe, Wolf-Dietrich, $0 Z$

Pégard, Nicolas, 03

Piechotta, G., 05

Pohl, Leon, 09

Pufe, W., 02

Pulwer, Silvio, OF

Qi, Yi, 00

Quenzer, Hans-Joachim, 09

Reiche, Manfred, OY

Ristic, Lj., OB

Rohling, Hanna, OC

Rudloff, D., 02

Sabry, Yasser M., OK, OT, OU, OV, OW

Sacher, Joachim R., OC

Saeed, Ahmed, OU

Sandner, Thilo, $\mathrm{OZ}$

Schanze, Thomas, OC 
Schenk, Harald, OD

Schenk, Hermann A. G., OD

Schmidt, J., 02

Schmidt-Grund, Rüdiger, OY

Schmidtmann, Sebastian, OC

Schrader, Sigurd, OF

Schröder, S., 04

Schroedter, Richard, 08

Schulze, M., 02

Schwarz, Fabian, 09

Schwarzenberg, Markus, $\mathrm{OZ}$

Senger, Frank, 05, 09

Shaheen, Amir K., OK

Shawkey, Heba A., OU

Shebl, Ahmed, OV

Spitz, Barbara, OD

Stolz, Michael, OD

Su, Yu, OB

Tatenguem, Herve, OC

Todt, Ulrich, 08

Torlee, $\mathrm{H} ., \mathrm{O} 2$

Uttamchandani, Deepak, OM

Villringer, Claus, OF

von Wantoch, Thomas, 05, 09

Wagner, B., 04

Wagner, M., 02

Wall, Franziska, OD

Waller, Laura, 03

Wildenhain, Michael, $\mathrm{OZ}$

Yalcin, Cem, 03

Yearim, G., OG

Yee, S., OS

Yehiel, M., OG

Young, J., OS

Zhou, Guangcan, 00

Zhou, Guangya, 00 


\section{Conference Committee}

Symposium Chairs

Sailing He, KTH Royal Institute of Technology (Sweden) and Zhejiang University (China)

Yasuhiro Koike, Keio University (Japan)

Symposium Co-chairs

Connie J. Chang-Hasnain, University of California, Berkeley (United States)

Graham T. Reed, Optoelectronics Research Centre, University of Southampton (United Kingdom)

Program Track Chairs

Holger Becker, microfluidic ChipShop GmbH (Germany)

Georg von Freymann, Technische Universität Kaiserslautern (Germany)

Conference Chairs

Wibool Piyawattanametha, King Mongkut's Institute of Technology Ladkrabang (Thailand) and Michigan State University

(United States)

Yong-Hwa Park, KAIST (Korea, Republic of)

Hans Zappe, University of Freiburg (Germany)

Conference Program Committee

Çağlar Ataman, University of Freiburg (Germany)

Robert Brunner, Ernst-Abbe-Hochschule Jena (Germany)

Pei-Yu Eric Chiou, University of California, Los Angeles (United States)

David L. Dickensheets, Montana State University (United States)

Jan Grahmann, Fraunhofer-Institut für Photonische Mikrosysteme (Germany)

Ulrich Hofmann, OQmented GmbH (Germany)

Ki-Hun Jeong, KAIST (Korea, Republic of)

Diaa Abdel Maguid Khalil, Si-Ware Systems (Egypt)

David G. Lishan, Plasma-Therm LLC (United States)

Veljko Milanović, Mirrorcle Technologies, Inc. (United States)

Yves-Alain Peter, Ecole Polytechnique de Montréal (Canada)

Zhen Qiu, Michigan State University (United States) 
Niels Quack, Ecole Polytechnique Fédérale de Lausanne (Switzerland)

Anna Rissanen, Outsight Oy (Finland)

Stefan Richter, Carl Zeiss AG (Germany)

Hamdi Torun, Northumbria University (United Kingdom)

Frédéric Zamkotsian, Laboratoire d'Astrophysique de Marseille (France)

Guangya Zhou, National University of Singapore (Singapore)

\section{Session Chairs}

1 Micro-mirrors

Çağlar Ataman, University of Freiburg (Germany)

2 LIDAR

Veljko Milanović, Mirrorcle Technologies, Inc. (United States)

3 Novel Optical Devices I

David G. Lishan, Plasma-Therm LLC (United States)

4 Novel Optical Devices II

Hans Zappe, University of Freiburg (Germany)

$5 \quad$ Imaging

Diaa Abdel Maguid Khalil, Si-Ware Systems (Egypt)

6 Endoscopic Microscopy: Joint Session with 11214 and 11293

Hans Zappe, University of Freiburg (Germany) 